\title{
Reforma tributaria amplia en El Salvador
}

\author{
Mark Gallagher \\ USAID/EI Salvador, ${ }^{1,2}$
}

La necesidad de llevar a cabo una reforma tributaria en El Salvador no fue reconocida por las autoridades gubernamentales sino hasta 1986. Durante el perlodo 1986-88, se hicieron cambios a la politica tributaria, pero estos no representaron una reforma amplia ni fueron acompañados por mejoras permanentes en la administración tributaria. Cuando la administración de Cristiani asumió el poder a mediados de 1989 , se agilizó la reforma tributaria, se le dio un enfoque más amplio y se preocupó no solamente por la generación de ingresos sino también por reducir las deficiencias económicas. Finalmente, en julio de 1992, la reforma de políticas tributarias alcanzó el pináculo con la aprobación del Impuesto al Valor Agregado (IVA). Además, con la asistencia técnica proporcionada por el gobierno de los Estados Unidos y el Banco Interamericano de Desarrollo, estas reformas tributarias están siendo acompañadas por reformas administrativas y programas de mejoramiento que, se espera, tendrán un impacto duradero sobre la capacidad del gobierno de El Salvador de administrar eficiente y equitatlvamente

1. Las opiniones y puntos de vista expresados en este documento son del autor, y no rellejan necesariamenle los de la Agencla para el Desarrollo Internacional.

2. El autor desea agradecer a Randall Peterson y Daniel Wisecarver por sus valiosas aportaciones. Todos los errores cometidos son, por supueslo, del autor. 
su nuevo y mejorado sistema tributario.

Al momento de redactar este documento, se puede decir que El Salvador posee en su cartera una de las estructuras tributarias económicas más eficientes en los paises desarrollados, y en algunas formas, su estructura tributaria es superior a muchos de los paises desarrollados,

Este documento enfoca las principales medidas tributarias, comenzando desde 1986, que precedieron a la reforma tributaria de 1991-92. Se discute también la necesidad que conllevó a una relorma tributaria más amplia en 1991-92 y su consecuente implementación. La magnitud de la reforma tributaria de El Salvador se presenta en un marco comparativo. Finalmente, el documento enloca los esfuerzos que se están haciendo para garantizar la capacidad administrativa del Gobierno de El Salvador para administrar eficiente y equitativamente su sistema tributario, y aquellos que se están haciendo para mejorar la capacidad analítica del Ministerio de Hacienda; un paso necesario para defender la reforma tributaria de acciones dañinas por intereses económicos especiales.

\section{Medidas tributarias anteriores}

Se tomaron varias medidas tributarias antes que se diese la reforma más amplia. Estas medidas aparentemente incluyeron un gran número de objetivos, algunos de los cuales contradictorios. Además, éstas no fueron tomadas dentro de un marco amplio de liberilización económica, sino por el contrario, en un contexto de intervenciones estatales considerables en la economia. Los bancos pertenecian al Estado; las lasas reales de interés fueron negativas para los sectores elegibles de recibir préstamos del sector bancario formal propiedad del gobierno; el tipo de cambio estaba controlado por el Banco Central; y la moneda estaba sobrevaluada. Algunos de los cambios realizados a la politica tributaria durante 1986-88 se resumen en los siguientes párrafos.

En octubre de 1986, la Asamblea Legislativa aprobó un impuesto único de emergencia sobre el capital (Soberania Nacional), desde $₫ 500$ más el 1 por ciento del capital sobre $₫ 100,000$ a $₫ 22,000$, más el 4 por ciento del capital sobre $₫ 1.0$ millón. Esta sobrecarga fue cambiada posteriormente, bajando la lasa máxima del impuesto marginal de 4 por ciento a 2.5 por ciento. Al mismo tiempo, sin embargo, el impuesto fue ampliado para incluir empresas como también activos personales. En febrero de 1987, la Corte Suprema decretó que este impueslo al capital era inconstitucional y el impuesto fue abolido.

La Asamblea Legislativa aprobó una serie de cambios más amplios a la política tributaria en diciembre de 1986, pero para que se ejecutasen 
hasta enero de 1987. Este conjunto de cambios a las políticas consistieron en 12 nuevas medidas, incluyendo: impuestos más altos sobre el ingreso personal, ingreso por negocios, patrimonio neto, cigarros, alcohol, tarifas aéreas y maritimas, tranferencias de los bienes muebles y registro de vehículos. Además, la sobrecarga de emergencia a las exportaciones de café fue reducida del 15 por ciento al 10 por ciento.

En 1987 y 1988, se emitieron decretos para cambiar la estruclura tributaria del pals. El impuesto sobre la renta fue modificado para incluir un tramo de impuesto cero por ingresos. Anteriormente, cualquier ingreso hasta $₫ 7,000$ estaba sujeto a un 2.85 por ciento de retención de impuestos; esto cambió para exonerar ingresos hasta $₫ 12,000$. Las tasas impositivas marginales individuales fueron cambiadas de un rango del 2.85 a 60.00 por ciento a 7.40 a 60.00 por ciento. Se aumentó un número de deducciones. Por ejemplo, la deducción por un dependiente fue aumentada de $\$ 1,599$ a $\$ 2,000$, aunque al mismo tiempo, el número de deducciones por dependiente fue reducido para aquellas personas cuyos ingresos eran altos.

El impuesto sobre la renta de personas naturales también fue enmendado. Las personas jurldicas estaban sujetas a un impuesto del 2.5 por ciento sobre ingresos hasta $₫ 10,000$. El nuevo decreto mandaba que los primeros $\$ 25,000$ de los ingresos empresariales estuviesen exentos de impuestos sobre la renta. Se aumentó el rango de'tasas impositivos marginales de 5.0-30.0 por ciento a 15.0-35.0 por ciento. Desde luego que muchos de los cambios, tanto en las estructuras Iributarias personales como de empresas, fueron necesarias debido a la rápida inflación. Por ejemplo, desde 1980-88, el índice de precios al consumidor habla aumentado en un 390 por cienlo acumulado.

De igual manera, se implementaron otros cambios tributarios. El impuesto a la transferencia de bienes muebles fue aumentado de una tasa fija del 0.5 por ciento a una variedad de tasas, oscilando desde el 1.5 por ciento a 5.0 por ciento por propiedades valuadas en más de $\$ 250,000$. El patrimonio neto (previamente llamado "viabilidad"), el tramo de impuestos por cero fue incrementado de $\$ 25,000$ a $\$ 150,000$ y el rango de las tasas marginales fue aumentado de $0.10-1.40$ por ciento a 0.35-2.50 por ciento. El impuesto sobre las tarilas aéreas y marítimas fue aumentado del 10 al 20 por ciento; el impuesto por consumo de cervezas fue aumentado de $\mathbf{4 2 . 5}$ por ciento a $\mathbf{4 5}$ por ciento; el impuesto sobre cigarros fue aumentado de $\mathbf{4 2 . 5}$ al $\mathbf{5 0}$ por ciento, y 100 por ciento a los cigarros extranjeros (el aumento del impuesto a los cigarros fue posteriormente anulado); el impuesto sobre el aguardiente fue aumentado de 2,32 a $\$ 2.70$ por litro; y el impuesto ad-valorem sobre licores fue 
aumentado de 25 a 30 por ciento.

Es difficil clasilicar estos cambios hechos a las políticas tributarias. Algunos parecen estar encaminados hacia la captación de mayores ingresos para el tesoro. Otros, para aumentar la progresividad del sistema tributario; por ejemplo, se aumentó el tramo de impuesto por cero para el impuesto sobre la renta mientras se reducian las deducciones permisibles para contribuyentes con ingresos más altos. Aparentemente, obtener mayor eficiencia económica no habla sido uno de los objetivos de eslos cambios en la polfica tributaria, puesto que al aumentar los impuestos a rubros especílicos de consumo probablemente tiene efectos opuestos. Tampoco se puede decir que estos cambios tributarios trataban de motivar el crecimiento económico por medio de ahorros y acumulación de capilal, ya que al aumentar las tasas impositivas sobre el patrimonio neto o ingreso empresarial parece proporcionar discentivos de ahorros e inversiones. En gran medida, estos cambios tributarios se dieron a ralz de la necesidad que el Gobierno de El Salvador tenia de captar mayores ingresos pero sin preocuparse del impacto económico de estos impuestos. Adicionalmente, aunque exista algún grado de interés en la equidad, estos cambios en las politicas aparentemente no trataron sistemáticamente de enfocar aspectos de equidad.

Después de asumir el poder en 1989, la administración de Cristiani introdujo una serie de políticas tributarias y cambios administrativos. Estas medidas tributarias fueron tomadas dentro de un marco de liberización de la economía salvadoreña. Las divisas podian ser ahora comercializadas desde un mercado mas libre y abierto, los bancos serían privatizados y las tasas de interés se aumentaron a mayores niveles económicos. El principal cambio tributario fue la simplificación del impuesto de timbres, reduciendo el número de bienes exentos y aplicando una sola tasa de cinco por ciento sobre las ventas, en lugar del rango anterior del uno al cinco por ciento.

Los cambios adicionales a la política tributaria eliminaron la mayoria de las exensiones, exoneraciones y otro tratamiento especial de los impuestos sobre la renta y los bienes importados. Además, la tasa máxima marginal del impueslo sobre la renta fue reducida del 60 por ciento al 50 por ciento; las tasas impositivas fueron simplificadas, reduciendo asl el número de tramos tributarios de 24 a 7 ; y el escalón tributario de cero fue aumentado de $₫ 12,000$ a $₫ 18,000$. La estructura de tasas impositivas sobre el ingreso de las personas jurídicas fue simplificada de cinco tramos oscilando entre el 15 por ciento y 35 por ciento, a tres tramos de 10 por cienlo, 20 por ciento y 30 por ciento.

En 1989, se realizaron varias visitas de inspección a las grandes 
empresas por los administradores y auditores fiscales, y los respectivos cierres mostraron al público la seriedad del gobierno en combatir la mora fiscal. Sin embargo, la ofensiva de noviembre de 1989 por el FMLN evitó estas visitas y el electo demostrativo de la disposición del gobiemo de reforzas la ley fiscal se vio reducida grandemente.

En 1990, varias medidas fueron adoptadas; algunas con el objetivo de mejorar la equidad en la incidencia tributaria y otras con la meta de reducir las distorciones económicas o facilitar la aplicación fiscal:

- Se eliminaron los derechos arancelarios sobre el camarón y la caña de azúcar.

- Los derechos de importación fueron reducidos de un rango de 0 a 290 por ciento a 5 a 50 por ciento, y el número de tarifas arancelarias fue reducido de 25 a 7 ; y se eliminaron muchas exenciones.

- Se introdujo un sistema de retenciones mensuales para el impuesto sobre la renta de personas jurídicas.

- Se concedió un aumento en las deducciones para los gastos médicos y educativos bajo el impuesto sobre la renta de personas naturales.

- Con el objeto de atraer mayores inversiones, se concedió a las empresas una deducción de hasta el 50 por ciento del ingreso imponible por reinversiones de utilidades.

- El gobierno promulgó una amnistía que permitió que los impuestos vencidos fuesen pagados con bonos del gobierno y sin multa.

Para 1991, el sistema tributario salvadoreño habla mejorado considerablemente, tanto desde la perspecliva de reducción de ciertos aspectos regresivos del impuesto sobre la renta como desde el punto de vista de mejoramiento de los incentivos económicos y reducción de las distorsiones. Sin embargo, con la pérdida de los ingresos por las exportaciones de café debido a los precios tan bajos y a la reducción en la producción, que hasla hace pocos años había proporcionado al gobierno de El Salvador casi un tercio de todos sus recibos fiscales, hubo necesidad de generar nuevos ingresos sin reincidir en los logros de equidad y eficiencia de años recientes.

\section{Reforma tributaria amplia}

La reforma tributaria en El Salvador se inició con algunos de los cambios a la anterior ley tributaria mencionada más arriba, cuyo avance no fue logrado sino hasta finales de 1991 y 1992. Se han tomado tres mecanismos de enfoques: 1) reducir la protección efectiva al cambiar el 
arancel; 2) reducir la complejidad del impuesto sobre la renta mientras se introduce mayor equidad y se reducen los discentivos; 3) substituir un impuesto de timbres diffcil de ejecutar por un impueslo de consumo con bases amplias (es decir, el impuesto al valor agregado). Las reformas al impuesto sobre la renta y a los derechos arancelarios, aparte de reducir las distorciones económicas, están diseñadas para reducir la evasión al simplificar las regulaciones y el número de tramos fiscales, $y$ al reducir los incentivos para cometer fraude. Aunque el impuesto al valor agregado no simplificará el cumplimiento, se espera que la documentación generada de su aplicación sí aumentará el cumplimiento.

Existe un número de razones para aplicar diferentes impuestos en lugar de ejecutar solamente uno. Por ejemplo, muchos de los países del tercer mundo encuentran que su capacidad de implementar impuestos sofisticados, tales como el impuesto sobre la renta o el IVA, se ve limitada severamente por la falta de personal ejecutor debidamente capacitado, normas contables pobres, incentivos bajos para cumplir las leyes tributarias por los oficiales gubernamentales, bajos niveles de eficiencia y otras razones. En tales casos, los países del tercer mundo a menudo confían, en gran medida, en los derechos arancelarios que son relativamente fáciles de captar puesto que los bienes ingresan al pais a través de pocos puertos de entrada, y debido a otros procesamientos que estas importaciones tienen que seguir. Por las mismas razones, los países impondrán impuestos sobre exportaciones especificas, aun cuando esto es menos común debido al estrago percibido para crear generación de divisas. Sin embargo, los exportadores de un solo bien o varios bienes, tal como El salvador que ha dependido del café para percibir la mayoria de su divisas, a menudo exige un impuesto de sus principales exportaciones.

El impuesto sobre la renta puede complementar otros tipos de ingresos resultantes de impuestos mientras se introduce mayor progresividad al sistema tributario general. Al mismo tiempo, ciertas deducciones de impuestos, créditos y otras reglas, a menudo son usadas para agilizar logros sociales específicos o para adoptar efectos externos de mercados especificos. Por ejemplo, los incentivos fiscales pueden motivar empresas a proporcionar capacitación a los trabajadores o invertir en industrias o regiones especificas del país.

Las medidas especificas tomadas en 1991 y 1992 que componen la reforma tributaria se discuten a continuación.

Impuestos comerciales: A principios de 1991, los derechos arancelarios fueron nuevamente reducidos de la principal sección del código arancelario con un arancel máximo del 35 por ciento, aun cuando el 
número de articulos que permanecieron fuera del principal código arancelario continuaban siendo tasados en base a tarilas mucho más altas. Durante el transcurso del año, la tasa tope de los articulos del código arancelario fue disminuida a 20 por ciento y del código extraordinario fue disminuida a 35 por ciento. Este nuevo cambio en los códigos arancelariós fue efectivo a partir del 7 de enero de 1992. Actualmente, la tasa máxima es de 30 por ciento y el GOES está analizando reducirla a 20 por ciento.

Impuestos sobre la renta: En diciembre de 1991, la Asamblea Legislativa aprobó una nueva ley que disminuyó de $\mathbf{5 0}$ por ciento a $\mathbf{3 0}$ por ciento la tasa máxima marginal para el impuesto sobre la renta personal. Esle nuevo código fiscal también estableció una tasa tributaria promedio máxima del 25 por ciento. Por lo tanto, aunque la tasa marginal más alta alcance hasta el 30 por ciento después de ciertos niveles de ingresos disminuya a 25 por ciento con una tasa promedio máxima del 25 por ciento. La ley del impuesto sobre la renta también aumentó la categoría de cero para ingresos personales hasta $\$ 22,000$ (casi $\$ 2,500$ en mayo de 1993) y para ingresos empresariales a $₫ 75,000(\$ 7,500)$.

Todos los ingresos imponibles de las personas jurídicas arriba de los $\$ 75,000$ serían tasados en base a una tasa común fija del 25 por ciento. La reforma de diciembre de 1991 también incluía que todas las empresas usaran el año calendario como su año fiscal. El propósito de este cambio era evitar que los empresarios ocultasen sus ingreso al transferir el precio y movilizar las ganancias entre sus negocios teniendo varios años fiscales.

Tanto el ingreso personal (naturales) como empresarial (jurídicas) lue completamente coordinado en mayo de 1992, para que el impuesto pagado sobre los ingresos por dividendo fuesen acreditados al ingreso personal de una persona. Esto eliminó la doble imputación de los ingresos y abolió distorciones en la progresividad al dar un tratamiento personal al ingreso en lugar de institucional.

Impuesto al Valor Agregado (IVA): El 29 de julio de 1992, la Asamblea Legislativa aprobó la ley que substituiría el impuesto de timbres y papel sellado por el del IVA. El papel sellado habla sido solamente una fuente tributaria menor pero el impuesto de timbres fue una de las más grandes fuentes de ingresos para el gobierno de El Salvador. El impuesto de timbres constiluyó un impuesto cascada con innumerable exenciones. La tasa nominal de timbres era del 5 por ciento pero si se reforzaba habría sido una tasa efectiva aproximadamente entre el 15-18 por ciento. EI IVA fue aprobado por la Asamblea Legislativa con una tasa de solamente el 10 por ciento, en lugar del 12 por ciento propuesto por el gobierno, cuya vigencia fue a partir del, $1^{2}$ de septiembre de 1992. 
Al comparar el IVA en El Salvador con el de otros paises, el IVA salvadoreño debe ser visto como un modelo para otros palses. La ley del IVA contiene muy pocas exenciones: leche, granos básicos, medicinas $\theta$ insumos médicos, educación y servicios de salud pública, agua y electricidad. Además, las exportaciones están "tasadas de cero", aunque las inversiones son generalmente tratadas como cualquier otro insumo de la producción.

\section{Capacidad administrativa}

La falta de personal capacitado y de normas contables, entre otras razones, ha conducido a una pobre implementación tributaria. El impuesto sobre la renta, el impuesto de timbres y los derechos arancelarios en EI Salvador, han producido en forma constante los bajos niveles de ingresos para el gobierno. La simplificación del impuesto sobre la renta y las tarifas arancelarias de las importaciones ayudan en la implementación de estos impuestos al reducir la necesidad de clasificar bienes importados en varias categorlas y al reducir la carga contable que requiere el cumplimiento del impuesto sobre la renta. Al mismo tiempo, al eliminar las deducciones del impuesto sobre la renta en base a lasas impositivas disminuidas dará como resultado menores ingresos del impuesto sobre la renta que tiene que ser captado por el tesoro, a menos que se implementen los pasos administrativos más amplios de la base fiscal. Mientras tanto, el implementar el IVA en lugar del impuesto de timbres implica no solamente mayores cargas sobre las empresas sino también sobre el personal gubernamental responsable de su ejecución.

En la actualidad, son varios los pasos que están en proceso de ejecución, tales como la política fiscal que está siendo reformada, con la expectativa de mejorar la capacidad del gobierno para administrar su sistema fiscal. La mayor parte de estos esfuerzos están siendo implementados con asistencia técnica ( $y$ alguna ayuda en bienes) proporcionada por el gobierno de los EE.UU., el Fondo Monetario Internacional y el Banco Interamericano de Desarrollo (BID).

Se ha diseñado un sistema para la administración del IVA, el cual puede producir reportes que permitirán a los administradores fiscales determinar si una industria o empresa especílica está pagando menos de lọ que está supuesta a pagar, si la industria o empresa está sometiendo el número apropiado de declaraciones, o si existen irregularidades en los reportes de ventas y compras. Los datos de este sistema pueden ser ligados a los datos de los rendimientos fiscales empresariales o personales, para obtener una base más completa y exacta, para determinar la mora y los errores fiscales. Hace dos años, sólo existla una unidad de- 
dicada a la ejecución del impuesto de timbres y otra dedicada a la ejecución del impuesto sobre la renta. Ambas unidades han sido ahora fusionadas, lo cual fortalecerá la capacidad del gobierno para usar información sobre el IVA y el impuesto sobre la renta en forma más coordinada. Además, se está proporcionando capacitación a los auditores fiscales, tanto para realizar auditorias al IVA como al impuesto sobre la renta.

La Organizaçión para el Desarrollo Industrial de las Naciones Unidas (UNIDO) ha estado proporcionando asistencia a la División de Aduanas de El Salvador para implementar un sistema de seguimiento de comercio (en español se denomina SIDUNEA). La asistencia de los Estados Unidos ayudará a mejorar la capacidad de Aduanas a fin de clasificar y valuar las importaciones por medio de un sistema computarizado que contiene una base de datos con los valores de los productos y una clasificación previa, incluyendo la aplicación de tasaciones. Se están haciendo otros esfuerzos para mejorar la administración de Aduanas, especialmente por medio de capacitación a los inspectores de Aduanas, desarrollo de un departamento de investigación y otros cambios más amplios en la política administrativa.

Se terminó recientemente un proyecto piloto que utiliza lecturas de tarjetas magnéticas para las cobranzas y reporte de los recibos fiscales, según se cobra en los bancos y en las cajas del gobierno. Estas lecturas de tarjelas magnéticas mejorarán la contabilización de los recibos fiscales, forzará la reconciliación entre los recibos y los diferentes depositarios de recolectar impuestos, y las cuentas de los contribuyentes y el tipo de impuesto de todos los recibidos diaria, mensual y anualmente. El proyecto ya ha sldo exlendido a nivel nacional.

\section{Amplitud de las reformas de EI Salvador}

Se ha dicho que las reformas fiscales de 1991 y 1992 han representado más bien reformas amplias del sislema fiscal en El Salvador. Esto merece una explicación.

La reforma fiscal recientemente llevada a cabo en El Salvador puede ser vista en forma amplia ya que comprende tanto impuestos directos como indirectos. Los impuestos que estas reformas enfocan incluyen casi el 90 por ciento de lodos los ingresos fiscales; y las reformas incluyen amplitud de la base, eficiencia económica y, en forma más limitada, la equidad. Los temas de equidad vertical están incorporados a las reformas del impuesto sobre la renta puesto que se ha introducido mayor progresividad por medio del aumento de la categorla de cero ingresos y se han eliminado muchas de las deducciones que solamente las personas más ricas podlan aprovechar. La equidad horizontal ha sido fortalecida 
principalmente al ampliar la base y al eliminar muchas exenciones, incluyendo una base más amplia para el impuesto de consumo (IVA), mientras que la simplificación general de las tarifas de importación y los impuestos sobre la renla garantizan un tratamiento más justo al reducir las oportunidades de fraude y evasión.

Otros palses menos desarrollados han tenido más o menos relormas amplias. El cuadro que se presenta a continuación compara estas reformas fiscales con las reformas tomadas en El Salvador.

\begin{tabular}{|c|c|c|c|c|c|}
\hline \multicolumn{6}{|c|}{ Tipo de reforma } \\
\hline Pais & rentas & comercio & ventas & $\begin{array}{l}\text { pro. } \\
\text { /patrim. }\end{array}$ & $\begin{array}{l}\text { adminis } \\
\text { trativa }\end{array}$ \\
\hline $\begin{array}{l}\text { El Salvador (91/2) } \\
\text { Chile (1974-79) } \\
\text { Colombia (1974)* } \\
\text { Indonesia (1983) } \\
\text { Jamaica (86/87) } \\
\text { Sri LAnka (78/88) } \\
\text { Uruguay (med. } 70 \text { ) }\end{array}$ & $\begin{array}{l}E, Q \\
E \\
E, Q, B \\
E \\
E, B \\
E \\
E\end{array}$ & $\begin{array}{l}\text { E,Q } \\
\text {.. } \\
\text { ninguna } \\
\ddot{E} \\
\text { X." } \\
\text {,E }\end{array}$ & $\begin{array}{l}\text { E,Q,B } \\
\text { E,Q,B } \\
\text { ninguna } \\
\text { E,Q,B } \\
\text { ninguna } \\
\text { R,B } \\
\text { E,Q,B }\end{array}$ & $\begin{array}{l}\text { ninguna } \\
\ddot{E} \\
\ddot{\text { ninguna }} \\
\mathbf{E} \\
\text { S }\end{array}$ & $\begin{array}{l}\text { sl } \\
\text { si } \\
\text { ninguna } \\
\text { si } \\
\text { ninguna } \\
\text { ninguna } \\
\text {.. }\end{array}$ \\
\hline \multicolumn{6}{|c|}{$\begin{array}{l}\text { Significado: } \\
E=\text { eficiencia, } Q=\text { equidad, } B=\text { ampl. de la base, } S=\text { cambio conlo Intención } \\
\text { claramenle identificable, } X D=\text { impuesto sobre export., } M=\text { impuesto sobre } \\
\text { import., } A=\text { ambiguo, } . .=\text { sin mencionar. }\end{array}$} \\
\hline \multicolumn{6}{|c|}{$\begin{array}{l}\text { Notes: } \\
\text { " Los años de 1974-86 presenciaron la regresión del sistema fiscal colombia- } \\
\text { no. } \\
\text { " La reforma fiscal de comercio debla ser implementada de 198-1992. }\end{array}$} \\
\hline \multicolumn{6}{|c|}{$\begin{array}{l}\text { Fuenles: Chile, Indonesia y Uruguay de Harberger (1989); Colombia de } \\
\text { McLure (1989b); Jamaica de Bahl (1989); Sr Lanka de Jenkins (1989). }\end{array}$} \\
\hline
\end{tabular}

De los paises del cuadro anterior, solamente Chile ha implementado reformas tan amplias como las de El Salvador, y la mayoría de los paises tomaron más tiempo en implementar sus programas de reformas menos radicales. La reforma fiscal de El Salvador fue ejecutada en poco más de dos años. Sri. Lanka, por otro lado, demoró diez años en implementar las reformas, las cuales no fueron tan amplias como las de El Salvador. Por ejemplo, el aspecto de eliciencia se encuentra en todo el 
programa de reformas de El Salvador, mientras que Sri Lanka aparentemente sólo enfocó la eficiencia como un objetivo claro en su reforma de tierra y del impuesto sobre la renta. Cuando consideramos que los ingresos del impuesto sobre la renta son solamente el cuatro porciento de los ingresos fiscales totales del gobierno, debemos también tomar en cuenta esa reforma con interés.

A finales de los años 70, Chile implementó un IVA. Este incluia tres objetivos: aumentar la equidad, reducir las distorciones económicas y ampliar la base fiscal. La reforma fue acompañada de reformas administrativas que asegurarían la administración eficiente del IVA. La reforma fiscal de Jamaica se ha estado ejecutando durante los últimos cuatro años, sin embargo, aun no representa la amplitud del programa de El Salvador. De todos los palses mencionados en el cuadro, se puede decir que solamente Indonesia ha implementado una reforma fiscal como la de EI Salvador y lo ha logrado en un perlodo de tiempo relativamente corto.

\section{Protección de las reformas}

¿Cómo se puede proleger la reforma fiscal de El Salvador? ¿Existen aspectos relativos a la relorma fiscal que puedan ser agregados al sostenimienlo? ¿Pueden las experiencias de otros paises darnos una respuesla a eslas preguntas?

Malcolm Gillis (1989) señala una serie de lactores que parecen haber sido importantes para asegurar el éxito a largo plazo de la reforma fiscal en paises menos desarrollados: 1) la falta de una crisis fiscal aguda garanliza que la reforma fiscal no necesita ser agilizada y presionada, y por ende, se puede disponer de suficiente tiempo para obtener una reforma bien diseñada; 2) la implementación del IVA para reemplazar un impuesto arcaico generalmente es irreversible (aunque a menudo puede ser moderado al incluir excepciones y tarifas variables); 3) una reforma fiscal amplia que también incluya reformas administrativas ayudará a garantizar el sostenimiento; y 4) la falta de tácticas fiscales, es decir, una reforma fiscal implementada sin confiar en estrategemas tales como loterias, supuestos impuestos agrícolas, o auto-evaluación del impuesto a la propiedad. ${ }^{3}$ En cada paso, la reforma fiscal de El Salvador parece haber evilado dificultades o haber tomado las iniciativas correctas. Aun

3. Gillis señala otros dos aspectos de programas fiscales exitosos que atañen a la duración del régimen en el poder después de ejecutadas las reformas y la consistencia de la política. Estos aspectos sólo pueden llegar a ser aparenles después de varios años de la reforma, y por ende, ser aun irrelevantes al caso salvadoreño. 
cuando el programa de reformas a la ley fiscal fue básicamente implementado en poco más de un año, y de hecho sin necesidad de gran cantidad de análisis sofisticados, fue un punto a favor para la plataforma política del partido que asumió el poder en 1989. Anteriormente, una reforma fiscal menos amplia involucró discusiones y análisis extensos. Indudablemente, para 1988, el Fondo Monetario Internacional ya habla recomendado una serie de medidas fiscales, incluyendo el IVA, y en 1990 , habla producido un plan completo para la implementación del IVA en EI Salvador.

Con el objeto de apoyar la reforma fiscal en El Salvador, se está proporcionando asistencia técnica al GOES para perfeccionar la administración, auditorla y cobranzas fiscales, y las operaciones del tesoro. Además, el Ministerio de Hacienda ha establecido una oficina de analistas de ingresos, que serán responsables del análisis cuantitativo de los ingresos fiscales, impactos de los cambios fiscales e incidencia fiscal. Este grupo de analistas podrá proporcionar asistencia intelectual en defensa de la reforma fiscal de El Salvador al indicar claramente los ganadores, perdedores, y las pérdidas y ganancias que podrían resultar de los cambios a la ley fiscal.

Una serie de factores adicionales se ajustan apropiadamente a la reforma salvadoreña. Primero, las reformas han sido amplias ya que han incluido tanto la reducción de distorciones económicas como reformas administrativas, aunque aun exisle mucho por lograr para mejorar la administración fiscal. Segundo, a pesar de la fuerte resistencia popular y política con respecto al impuesto al valor gregado, no hay un marcado interés por parte de algún grupo que presione la restauración del impuesto de timbres. Por lo tanto el IVA parece que es irreversible. Sin embargo, esto no implica que el IVA en forma más bien simple no se verá amenazado por intereses propios que tratarán de imponer exenciones a las industrias, produclos, grupos sociales y fases de producción, $y$ asI complicar y distorcionar el impuesto al consumo. Tercero, el GOES ha sido afortunado, ya que aún cuando la reforma fiscal ha sido implementada bajo condiciones de un desarrollo fiscal bastante pobre, especialmente debido a los precios bajos internacionales del café, se ha mantenido el apoyo dado por el gobierno de los EE. UU. al presupuesto ordinario, disminuyendo aśl la presión del aparato fiscal.

\section{Bibllografía}

Bahl, Roy (1989) "The Politlcal Economy of the Jamaican Tax Peform," in Gillis (1989a).

Cnossen, Sijbren (1992) "Key Questions in considering a Value-Added Tax lor 
central and Eastern European countries," International Monetary Fund Staff Papers Vol. 39, №2 2, June.

Gillis, Malcolm ed. (1989a) Tax Reform in Developing Countries, Duke University Press: Durham and London.

Gillis, Malcolm (1989b) "Tax Peform: Lessons form Postwar Experience in Devleoping Nalions," in Gillis (1989a).

Jenkins, Glenn P. (1989) "Tax changes belore Tax Policies: Sri Lanka, 1988-88," in Gillis (1989a).

Harberger, Amold (1989) "Lessons of Tax Reform from the Experiences of Uruguay, Indonesia, and Chile," in Gillis (1989a).

Intemational Monetary Fund (1988) El sistema Tributario de El Salvador y su Administración, prepared by Peter S. Griffith and gonzalo Sepulveda, December 5.

International Monetary Fund (1990) Una IVA para El Salvador, prepared by Isaaias Coelho, Jaime Ross and Joao J. Amaral Tomas, December 14.

McLure, Charles E. (1989) "Analysis and Reform of the colombian Tax system," in Gillis (1989a).

Shalizi, Zmarak and Wayne Thirsk (1990) Tax Reform in Malawi, World Bank Staff Working Paper No. 493, August. 